\title{
LA INNOVACIÓN ABIERTA COMO ELEMENTO DE ANÁLISIS EN LAS PEQUEÑAS Y MEDIANAS INDUSTRIAS. CASO SECTOR METALMECÁNICO
}

\author{
Anabel Terán Rojas \\ Universidad Nacional Experimental Politécnica “Antonio José de Sucre” V/R de Barquisimeto, \\ Venezuela \\ kdteran@yahoo.es
}

Carlos Rodríguez Monroy

Universidad Politécnica de Madrid, España

crmonroy@etsii.upm.es

\section{Nunziatina Bucci Peluso}

Universidad Nacional Experimental Politécnica “Antonio José de Sucre” V/R de Barquisimeto, Venezuela

nunziatinabucci@ hotmail.com

\section{RESUMEN}

La innovación abierta parte de la idea de que las empresas ya no son capaces de abordar todo el proceso de esta por sus propios medios y es un paradigma que asume que las organizaciones pueden y deben usar ideas externas, así como las ideas internas. El objetivo de la presente investigación es identificar las prácticas de gestión en el proceso de innovación abierta en las pequeñas y medianas industrias (PYMIs) en economías emergentes. La metodología se basó en una investigación de campo y la técnica de recolección de datos utilizada fue la entrevista, apoyada en un cuestionario (centrado en las Normas Españolas UNE 166002), cuyas categorías fueron: compromiso de la dirección, enfoque a las partes interesadas, recursos humanos y actividades de investigación, desarrollo e innovación. Participaron 30 industrias, resultando como elemento fundamental para la incorporación de la innovación abierta el compromiso a nivel gerencial de este sector industrial, además de mejorar los canales de comunicación con el entorno, consolidar la cultura de la innovación potenciando el trabajo en equipo, la motivación y la capacitación de recursos humanos y considerando las fuentes tanto internas como externas.

Palabras clave: Innovación abierta; Sector metalmecánico; Economías emergentes; Pequeñas y medianas industrias (PYMIs). 


\section{INTRODUCCIÓN}

Las naciones más avanzadas en la búsqueda del desarrollo, siempre han enfatizado en la actividad industrial realizada por las pequeñas y medianas industrias, por cuanto representan la actividad que permite una mayor productividad en las economías industrializadas. De hecho, su efectividad va mucho más allá de los países desarrollados, ya que han contribuido significativamente con el crecimiento económico de los países en desarrollo (Morillo, 2005).

A su vez, frente a la globalización de los mercados las industrias han considerado que su éxito o fracaso dependerá de la agilidad que tengan para dar respuesta a las oportunidades y amenazas del mercado. Las empresas innovan en productos y en procesos para mejorar sus resultados y obtener ventajas competitivas, considerando que los productos nuevos ayudan a captar y retener cuotas de mercado y mejoran la rentabilidad de esos mercados y los productos establecidos pueden competir en función del precio, el diseño, la adaptabilidad al cliente y la calidad. Además, la innovación en los procesos ejerce una labor igualmente importante y estratégica, ser capaz de hacer algo que nadie más puede hacer, o hacerlo mejor que el resto de las industrias, es una fuente poderosa de ventaja competitiva.

Es entonces, cuando se presenta la innovación abierta, la cual toma en cuenta una mayor cantidad de fuentes de innovación, y su origen cada vez más heterogéneo. La universalización en el acceso a internet ha revolucionado este campo: no sólo ha habido un proceso de movimiento off-shore de migración de la innovación desde los países desarrollados hacia los emergentes motivado por el encarecimiento de la innovación propia, también se ha dado el proceso a la inversa (Álvarez Garcia, 2009).

En este sentido, en los países en vías de desarrollo se están generando investigaciones orientadas al estudio de las PYMIs en el ámbito de la innovación abierta, lo cual permite determinar sus particularidades. Asimismo, el proceso de innovación en las organizaciones tiene características propias y diferenciables que se deben tener en cuenta (Asociación Española de Normalización [AENOR], 2006). De allí la importancia de estudiar sectores de la economía, para focalizar las necesidades propias de cada sector, y en este caso particular nos ocupa el sector metalmecánico.

El sector metalmecánico incluye a todas las empresas manufactureras que se dedican a la fabricación, reparación, ensamblaje y transformación de metales y es sin duda uno de los sectores fundamentales para la economía de los países industrializados y motor de desarrollo indispensable para

Revista de Administração e Inovação, São Paulo, v. 8, n. 2, p. 05-28, abr./jun. 2011 
los países emergentes, por su capacidad de transformar las materias primas en "instrumentos" industriales (Armenti, 2006).

Debe señalarse que, en los países latinoamericanos y más específicamente en Venezuela, la industria ferrominera, siderurgia y metalmecánica se integran en un único proceso: el del acero, a través de cuatro fases. La primera incluye las empresas que extraen el hierro y lo transforman en un aglomerado. La segunda se refiere al sector que produce hierro por reducción directa (HRD). La tercera está integrada por empresas que producen acero crudo, semielaborados, acero en láminas, y por último, la cuarta fase está constituida por las empresas del sector de la industria metalmecánica ferrosa, el cuál es objeto de este estudio.

Las tres primeras etapas se concentran en las industrias metalmecánicas pesadas (transformadoras de grandes cantidades de hierro), siendo además el centro neurálgico del cual dependen las materias primas. Sin embargo, la cuarta etapa representada por la industria metalmecánica que se ubica en el norte del país, no logra transformar en producto terminado toda la materia prima producida en Venezuela, que termina siendo exportada en su mayoría, por lo cual se desaprovecha esta ventaja. La producción venezolana se limita a hilos de hierro, aluminio, compresores, instrumentos hidráulicos, calderas, estructuras metálicas, fundiciones y moldes, válvulas, conexiones y también repuestos para automóviles.

Según el Consejo Nacional de Promoción de Inversiones (CONAPRI, 2006), en Venezuela esta actividad productiva aporta 45 por ciento del PIB del sector manufacturero, 42 por ciento del total de las exportaciones no tradicionales (2.800 millones de dólares) y el sector está conformado por más de 80 por ciento de empresas consideradas como pequeñas y medianas industrias (PYMIs), ocupando más de 45.000 trabajadores.

Este artículo presenta los resultados de una encuesta, asociada a una tesis doctoral, sobre el proceso de innovación en 30 industrias metalmecánicas, basada en la norma española UNE 166002 correspondiente a la Gestión de la I+D+i: Requisitos del Sistema de Gestión de I+D+i. El objetivo de esta investigación es una primera identificación de las prácticas de gestión habituales en el proceso de innovación abierta en este sector industrial, considerando que la innovación es un pilar fundamental para medir el espíritu competitivo mundial (Sala-i-Martin et al., 2007).

Los resultados obtenidos también permiten, por un lado identificar áreas de mejoras en el proceso de innovación abierta, y por el otro evidenciar la necesidad de introducir elementos de reflexión en el área gerencial con el fin de fomentar prácticas que permitan una respuesta flexible y de adaptación a los cambios. 
Asimismo, otro elemento que realza la importancia de esta investigación es poder contribuir con lineamientos para el establecimiento de políticas en el campo de la innovación. Tal como lo señala Asheim (2009), las políticas de innovación deben ser afinadas correctamente para que tengan en cuenta las respectivas estructuras industriales, y los entornos y sistemas sociales e institucionales.

El artículo está estructurado en seis apartados, introducción, innovación abierta, metodología, resultados y análisis, conclusiones y referencias bibliográficas.

\section{LA INNOVACIÓN ABIERTA}

En el Manual de Oslo, se define la innovación como la introducción de un producto nuevo o significativamente mejorado (bien o servicio), un proceso, un nuevo método de comercialización, un nuevo método organizativo, en las prácticas internas de la empresa, organización del lugar de trabajo o relaciones exteriores (Organización de Cooperación y Desarrollo Económico [OCDE], 2006).

Sin embargo, la innovación abierta desarrollada esencialmente en el ámbito empresarial, propone la colaboración, la co-creación con diferentes grupos de interés y la implicación de un esfuerzo interorganizativo y sinérgico, de otras organizaciones con misiones similares, comprometidas con el territorio o incluso con finalidades mixtas lucrativas y no lucrativas en el proceso innovador, que permita integrar el conocimiento interno y el externo (Chesbrough, 2009).

A su vez, Iturbe-Ormaetxe (2008) señala que es un concepto que maneja un pequeño grupo de autores, con diversidad de enfoques y que se encuentra en fase inicial de definición. Asimismo, está inducida por la sociedad red y ésta considera que las probabilidades de innovar son mayores tomando en cuenta el entorno y no solamente el talento interno de la organización. Se reafirma entonces, que al salir de las instituciones se evitan las limitaciones mentales y organizativas.

Según Chesbrough (2006), pionero en esta concepción, la innovación abierta es un paradigma que asume que las organizaciones pueden y deben usar ideas externas así como las ideas internas.

El concepto de innovación abierta parte de la idea de que las empresas ya no son capaces de abordar todo el proceso de la innovación por sus propios medios. Siendo relevante que cuenten con recursos externos (ideas, propiedad intelectual, productos e instituciones) y los integren a su propia cadena de innovación. Igualmente, los resultados de su trabajo pueden ser útiles para otras empresas u otros mercados, lo que constituye una manera de rentabilizar aquella innovación que resulta fallida para los objetivos primarios de la empresa (Álvarez Garcia, 2009).

Revista de Administração e Inovação, São Paulo, v. 8, n. 2, p. 05-28, abr./jun. 2011 
La última tendencia en organización empresarial, dentro del marco de este paradigma, es el crowdsourcing. Edwards (2008), señala que es otra forma de emplear mano de obra barata en la que cualquier persona, sin ser especialista, resuelve problemas para todas clases de compañías. Agrega, asimismo, que con las tecnologías de la información y comunicaciones (internet) es posible que las organizaciones tengan a su disposición el potencial de los millones de cerebros de la multitud que se conecta a través de la red. Al igual que el outsourcing, el crowdsourcing también origina ahorros económicos a las organizaciones, pero con la particularidad de que no importa la ubicación del talento o trabajador.

El modelo propuesto por Chesbrough (2009), se basa en la utilización de la base tecnológica interna e incorpora la base tecnológica externa, entre ellos las ideas, las capacidades, las tecnologías y los recursos provenientes de fuentes externas a la compañía. Tal como lo establece Bax (2008), muchas de las prácticas expuestas en este paradigma no son nuevas; sin embargo, agruparlas y organizarlas en un sistema lógico y coherente sí es nuevo y aporta una visión renovada de la gestión de desarrollo de negocio e I+D+i.

Pero bien, lo que hoy está sucediendo es un cambio significativo en el proceso y creación de productos. Se ha comenzado a integrar a los usuarios en la cadena de valor demostrando que sus demandas ahora pueden ser trabajadas por ellos mismos. De esta forma, las ideas geniales pueden formarse en cualquier dirección de manera abierta, logrando una retroalimentación a través de los mismos usuarios. Se genera entonces, un cambio de paradigma considerando que estamos abriendo nuestro contexto como organización hacia fuera, y a su vez el conocimiento será compartido y mejorado nutriendo y potenciando un proceso (que nos es lineal) a través de diversas miradas (Frías Rivas, 2008).

Entre los beneficios de la innovación abierta, reportados por Ayneto (2007) se encuentran: es una puerta abierta a nuevas ideas, sin estar sujetas a la cultura organizacional, estimula la innovación interna y genera sinergias, reduce y controla los costos de la innovación, se pueden obtener fácilmente resultados de calidad, se comparten riesgos y las nuevas ideas son fruto de múltiples experiencias.

Adicionalmente, para la aplicación de la innovación abierta Arias (2008), expone siete claves para su aplicación: aprovechar el mercado, acercarse al mundo de la investigación, adoptar estándares y regulaciones, explotar la propiedad intelectual, cultivar a las personas, moverse - hablar y escuchar, y por último, colaborar. 


\section{METODOLOGÍA}

La presente investigación, según los objetivos planteados, y de acuerdo a la clasificación presentada por Balestrini (2006) es de carácter descriptivo, ya que infieren la descripción con gran precisión acerca de las singularidades de una realidad estudiada. Este trabajo particular se centrará en la praxis del proceso de innovación abierta de las PYMIs metalmecánicas.

El diseño de la investigación en el que se basó este estudio es la investigación de campo, ya que se analizan de forma sistemática problemas de la realidad, con el propósito de describirlos, interpretarlos y entender sus factores constituyentes (Universidad Pedagógica Experimental Libertador [UPEL], 2006).

Como técnica de recolección de datos se utilizó la entrevista, apoyada en un cuestionario estructurado centrado en las Normas UNE 166002, el cual se basó en las siguientes categorías:

Compromiso de la dirección: comunicar la importancia de las actividades de $\mathrm{I}+\mathrm{D}+\mathrm{i}$, establecer la política de I+D+i, establecer los objetivos de $\mathrm{I}+\mathrm{D}+\mathrm{i}$, crear el departamento de I+D+i, asegurar la disponibilidad de recursos materiales, aprobar y revisar el presupuesto de $\mathrm{I}+\mathrm{D}+\mathrm{i}$, establecer la política de protección y explotación de los resultados.

$>$ Enfoque a las partes interesadas: atender la demanda de los proveedores y clientes, la motivación de los empleados, la demanda de los dueños, los requisitos legales y las innovaciones y cambios tecnológicos requeridos por el mercado.

Recursos Humanos: promover la toma de conciencia de la importancia de la I+D+i, motivar al personal, impulsar la participación de todo el personal, fomentar la creatividad y el trabajo en equipo, simplificar y facilitar las aportaciones de información de los distintos departamentos.

Actividades de $I+D+i$ : Este aspecto se subdividió en cinco subcategorías, a saber:

a) Subcategoría herramientas: utilizar vigilancia tecnológica, utilizar previsión tecnológica, utilizar la creatividad, utilizar el análisis externo e interno. 
b) Subcategoría acciones: identificar y analizar los problemas y oportunidades, analizar y seleccionar las ideas de I+D+i, planificar, seguir y controlar la cartera de proyectos, utilizar la transferencia de tecnología.

c) Subcategoría producto $I+D+i$ : concretar las ideas en un diseño básico, completar los detalles del diseño antes de las pruebas, crear un prototipo (prueba piloto), rediseñar, demostrar y producir, comercializar y documentar todos los cambios durante el proceso.

d) Subcategoría compras: asegurar que los proveedores satisfacen las necesidades del departamento de $\mathrm{I}+\mathrm{D}+\mathrm{i}$ y verificar las compras para garantizar que se cumple con los requisitos especificados

e) Subcategoría resultados del proceso de $I+D+i$ : documentar los resultados del proceso de $\mathrm{I}+\mathrm{D}+\mathrm{i}$ y proteger y explotar los resultados de las actividades de $\mathrm{I}+\mathrm{D}+\mathrm{i}$.

Para cada uno de los ítems del cuestionario, se solicitó la percepción a los gerentes entrevistados sobre el grado de cumplimiento en la industria objeto de estudio en una escala tipo Likert cuya estructura fue de la siguiente manera: (5) Excelente, (4) Bueno, (3) Suficiente, (2) Insuficiente y (1) Deficiente.

\subsection{VALIDEZ Y CONFIABILIDAD DEL INSTRUMENTO}

La validez del instrumento se determinó a través de una técnica recomendada por Palella y Martins (2003), la técnica del juicio de experto, donde tres expertos revisaron el contenido, la redacción y la pertinencia de cada reactivo y posteriormente se hicieron los ajustes necesarios.

La confiabilidad constituye la ausencia de error aleatorio en un instrumento de recolección de datos. Para esta investigación se utilizó el coeficiente alfa de Cronbach. En la tabla 1 se presentan los resultados del análisis de confiabilidad. 
Tabla 1 - Resultados del análisis de confiabilidad

\begin{tabular}{l|l|c|c}
\hline \multicolumn{4}{c}{ Resumen del procesamiento de los casos } \\
\hline Casos & Válidos & $\mathrm{N}$ & $\%$ \\
\hline & Excluidos & 6 & 80 \\
\hline & Total & 30 & 20 \\
\hline
\end{tabular}

Eliminación por lista basada en todas las variables del procedimiento.

\begin{tabular}{c|c}
\hline \multicolumn{2}{c}{ Estadísticos de fiabilidad } \\
\hline Alfa de Cronbach & $\mathrm{N}$ de elementos \\
\hline 0,978 & 81 \\
\hline
\end{tabular}

Fuente: Preparado por los autores

Con el resultado anterior, se revela que la consistencia interna de los ítems es muy alta (considerándose alta en el rango de 0.81 - 1, según Palella \& Martins, 2003), por tanto existe una alta confiabilidad. A su vez, es importante resaltar que los casos excluidos (señalados en la tabla 1), en el análisis de confiabilidad, corresponden a las empresas que dejaron al menos un ítem en blanco en el cuestionario.

\section{RESULTADOS Y ANÁLISIS}

\subsection{DESCRIPCIÓN DE LA MUESTRA}

Las PYMIs metalmecánicas (en un 67,86 \%) se dedican a la fabricación de piezas mecánicas, fundición y moldes, fabricaciones varias (máquinas hidrolimpiadoras, mobiliario para panadería, bombas mecánicas, pinturas electrostáticas y partes en goma), estructuras, equipos agrícolas, alambres, ganchos y mallas y tuberías y accesorios. Asimismo, el 75,36\% de las industrias del sector se dedican principalmente a tres procesos de transformación: operaciones de mecanizado, carpintería metálica y tratamientos térmicos (Bravo, Suárez, Terán \& Sánchez, 2008).

En esta investigación, se asume la clasificación propuesta por el Instituto Nacional de Estadística venezolano (Instituto Nacional de Estadística [INE], 2004), especificando que las pequeñas industrias poseen entre 5 y 20 trabajadores, la mediana industria inferior entre 21 y 50 trabajadores, la mediana industria superior tienen entre 51 y 100 trabajadores y la gran industria más de 100 trabajadores. 
Por otro lado, y a efectos descriptivos, se considera una muestra grande cuando $n>30$. Tal como lo señala García Muñoz (2005), una muestra debe ser lo suficientemente grande como para ser representativa, pero el número de elementos necesarios para lograr la representatividad varía de una investigación a otra. Agrega además, que cuanto más homogénea es una población en las características objeto de estudio, más fácil resulta obtener muestras representativas sin necesidad de que sean grandes.

En base a ello, y considerando que la confiabilidad es una propiedad del cuestionario para un grupo particular de sujetos y que depende de la homogeneidad del grupo, como el resultado de la confiabilidad en esta investigación fue muy alto, a partir de una población de 73 pequeñas y medianas industrias metalmecánicas, nos permitimos definir un tamaño muestral de 30 industrias, correspondientes al 41,09\% de la población, que se mostraron dispuestas a participar en el estudio.

De esta manera, el cuestionario se dirigió a los gerentes o directores, por el hecho de reconocer que en la mayoría de las industrias no está formalizado el departamento de I+D+i (Bravo et al., 2008). De hecho, el $87 \%$ fue respondido por los gerentes y el $13 \%$ lo respondieron personas que no ocupan cargos gerenciales (asistentes del área de producción y del área de proyectos).

\subsection{PROCESO DE I+D+I}

\subsubsection{Compromiso de la dirección}

Al evaluar el compromiso de la dirección, el ítem relativo a la disponibilidad de los recursos materiales se encuentra entre excelente y suficiente en el 70.37 por ciento de las industrias estudiadas. Asimismo, se comunica la importancia de las actividades de I+D+i en más del 62 por ciento y son insuficientes o deficientes los elementos correspondientes a la creación del departamento y los objetivos correspondientes a esta materia, al igual no se generan esfuerzos en establecer políticas tendientes a proteger y explotar los resultados obtenidos (ver tabla 2).

Sin embargo, y a pesar de no tener formalizado el departamento de $\mathrm{I}+\mathrm{D}+\mathrm{i}$, se analiza un presupuesto para ser destinado a la I+D+i que es canalizado por otros departamentos. 
Tabla 2 - Resultados de la evaluación de la categoría: compromiso de la dirección.

\begin{tabular}{l|c|c|c}
\hline \multicolumn{1}{c|}{ Aspecto } & $\begin{array}{c}\text { Excelente/ } \\
\text { Bueno } \\
\%\end{array}$ & $\begin{array}{c}\text { Suficiente } \\
\%\end{array}$ & $\begin{array}{c}\text { Insuficiente/ } \\
\text { Deficiente } \\
\%\end{array}$ \\
\hline $\begin{array}{l}\text { Comunicación de la importancia de las actividades de } \\
\text { I+D+i }\end{array}$ & 22.22 & 40.74 & 37.04 \\
\hline Establecer la política de I+D+i & 7.41 & 29.63 & 59.26 \\
\hline Establecer los objetivos de I+D+i & 18.52 & 18.52 & 62.96 \\
\hline Crear el departamento de I+D+i & 14.81 & 18.52 & 66.67 \\
\hline Asegurar la disponibilidad de recursos materiales & 40.74 & 29.63 & 29.63 \\
\hline Aprobar y revisar el presupuesto de I+D+i & 29.63 & 18.52 & 51.85 \\
\hline $\begin{array}{l}\text { Establecer la política de protección y explotación de los } \\
\text { resultados }\end{array}$ & 14.81 & 18.52 & 62.96 \\
\hline
\end{tabular}

Fuente: Preparado por los autores.

En el gráfico 1 puede observarse la representación de las medias para cada ítem correspondiente a esta categoría y la media más alta la representa el ítem correspondiente a la disponibilidad de recursos materiales, por lo que los empresarios están comprometidos y le dan prioridad a asegurar este recurso dentro de la organización, mientras que la creación del departamento de I+D+i no está formalizada dentro de la mayoría de las industrias y además, muchos entrevistados manifestaron no tener la necesidad inmediata de su constitución.

Por otro lado, se observa que la media de seis de los siete aspectos estudiados están por debajo del nivel suficiente, hecho que llama poderosamente la atención, ya que el compromiso de la dirección es de suma relevancia para que el proceso de I+D+i sea efectivo y tenga la fluidez. 


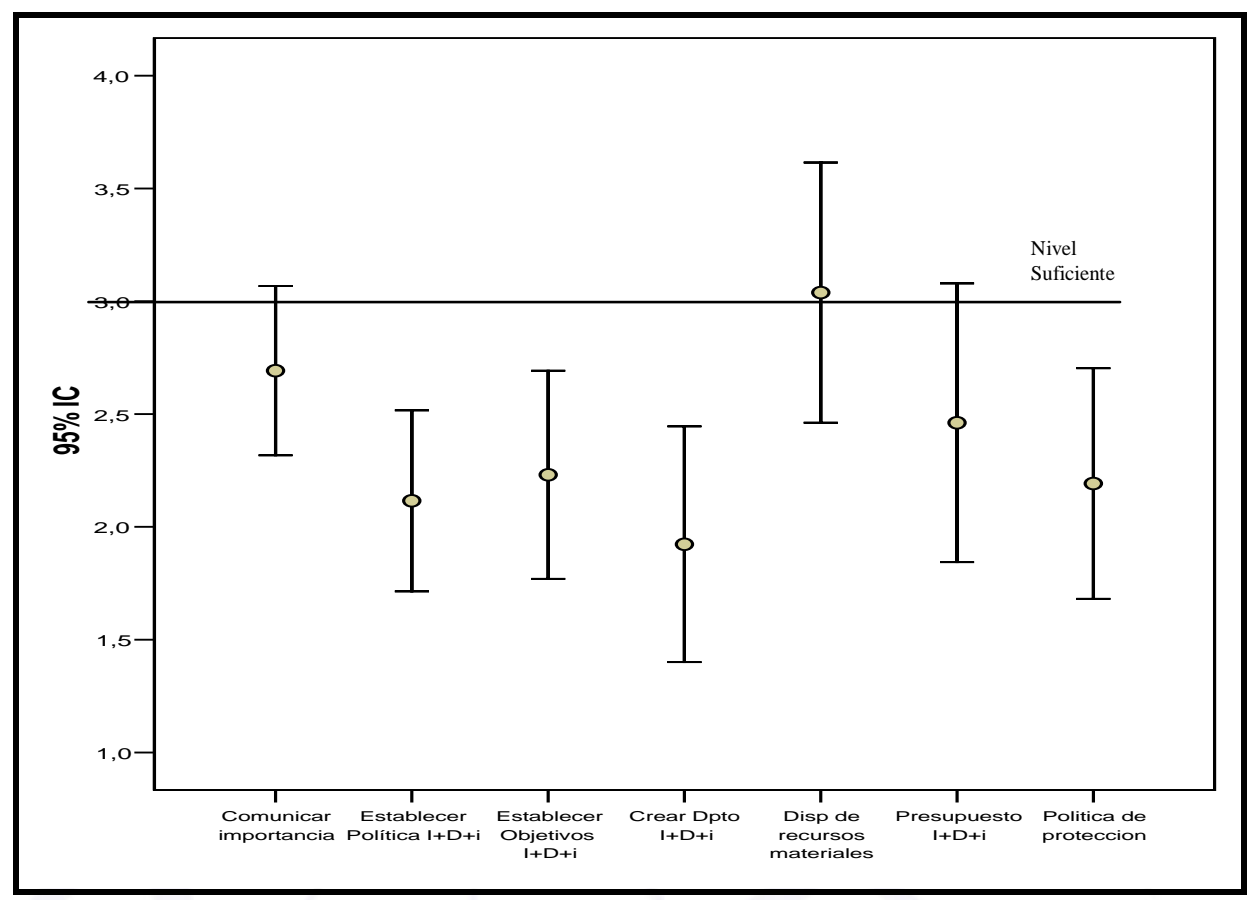

Gráfico 1 - Medias de los ítems correspondientes a: compromiso de ladDirección. Fuente: Preparado por los autores.

De esta forma se aprecia una brecha entre el resultado en esta categoría y el deber ser. Muchos gerentes están conscientes que la dirección, debe tener un gran sentido de compromiso con el sistema de gestión de I+D+i, para lograr su desarrollo y eficaz implantación y al mismo tiempo, que la innovación abierta traspasa los estrechos límites de un departamento, y se abre no solo a todo los miembros de la organización sino incluso a agentes externos, pero aún así hay escepticismo sobre la implantación de un sistema de innovación.

Igualmente, se debe tomar en cuenta que, el elemento central de cualquier proceso de innovación lo constituye la dirección, siendo la responsable de la obtención de un resultado aceptable bajo unas limitaciones de tiempo y de recursos (Hidalgo Nuchera, Vizán Idoipe \& Torres, 2008). Por tanto, el objetivo de la dirección de la innovación es la creación de condiciones internas en la empresa que contribuyan a la generación de procesos de innovación y a su finalización con éxito, considerando las fuentes tanto internas como externas.

\subsubsection{Enfoque a las partes interesadas}

Las necesidades y expectativas de los proveedores, clientes, empleados y dueños deben ser consideradas y analizadas por la dirección en el proceso de I+D+i, al igual que los requisitos legales, las innovaciones y los cambios tecnológicos requeridos por el mercado. 
En el caso del sector metalmecánico, la atención a las partes interesadas evaluadas, se encuentra en el rango de bueno/excelente, resultado significativo dentro del proceso de I+D+i. Sin embargo, la atención al mercado, en función de las innovaciones y los cambios tecnológicos es el ítem valorado con la menor puntuación en esta categoría, es decir, su percepción es insuficiente o deficiente en un 22 por ciento, por tanto, debe ser objeto de atención considerando que la innovación es un factor clave para la obtención de ventajas competitivas (Longanezi, Coutinho \& Bomtempo, 2008).

Tabla 3 - Resultados de la evaluación de la categoría: Enfoque a las partes interesadas.

\begin{tabular}{l|c|c|c}
\hline Aspecto & $\begin{array}{c}\text { Excelente/ } \\
\text { Bueno } \\
\%\end{array}$ & $\begin{array}{c}\text { Suficiente } \\
\%\end{array}$ & $\begin{array}{c}\text { Insuficiente/ } \\
\text { Deficiente } \\
\%\end{array}$ \\
\hline Atender la demanda de los proveedores y clientes & 88.89 & 7.41 & 3.70 \\
\hline Atender la motivación de los empleados & 62.96 & 29.63 & 7.41 \\
\hline Atender la demanda de los dueños & 77.78 & 3.70 & 7.41 \\
\hline Atender los requisitos legales & 88.89 & 7.41 & 3.70 \\
\hline $\begin{array}{l}\text { Atender las innovaciones y cambios tecnológicos } \\
\text { requeridos por el mercado. }\end{array}$ & 48.15 & 29.63 & 22.22 \\
\hline
\end{tabular}

Fuente: Preparado por los autores

Al representar las medias de los ítems referente al enfoque de las partes interesadas (gráfico 2) se aprecia que todos los elementos evaluados están por encima del nivel suficiente, además se refleja que el ítem correspondiente a la atención al mercado con respecto a las innovaciones y cambios tecnológicos está por debajo de la media del resto de los aspectos evaluados. 


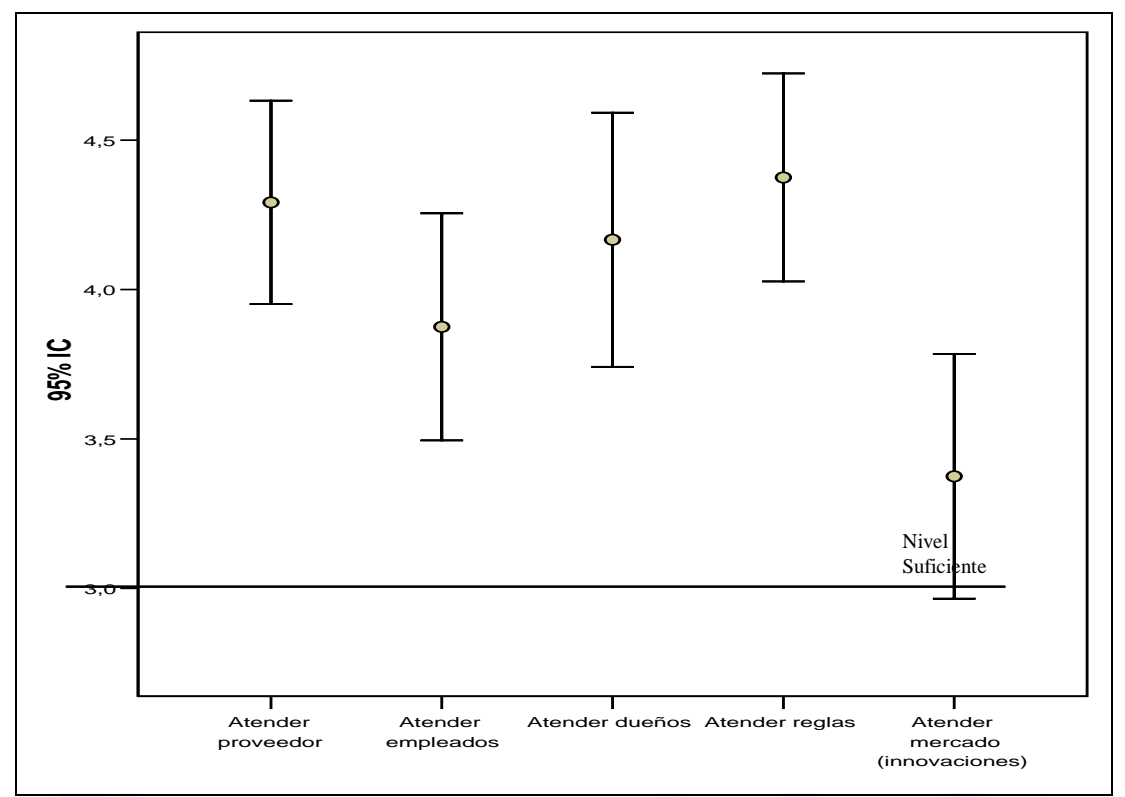

Gráfico 2 - Medias de los ítems correspondientes a: Enfoques a las partes interesadas.

Fuente: Preparado por los autores

En esta categoría, como se explicó antes, la debilidad de las empresas se encuentra en el ítem correspondiente a la atención a las innovaciones y cambios tecnológicos requeridos por el mercado. En este sentido, estas organizaciones necesitan incrementar los esfuerzos para potenciar las actividades de innovación referidas al mercado, en virtud que de nada sirve conocer las necesidades de sus clientes y de sus propios procesos operativos, si no se actúa en función de los mismos, y se pueden basar en el mejor uso tanto en las ideas internas como en las externas.

\subsubsection{Recursos Humanos}

Las industrias analizadas revelaron motivar al personal y fomentar la creatividad y el trabajo en equipo, hechos que favorecen el proceso de $\mathrm{I}+\mathrm{D}+\mathrm{i}$; sin embargo, sería de una forma más contundente si lo logran con todo el personal, ya que según los datos suministrados es impulsada de forma insuficiente/deficiente en un 44 por ciento (ver tabla 4). Asimismo, se debe tomar en cuenta que la clave del éxito de la I+D+i es la habilidad del personal para trabajar en equipo y su motivación para llegar a resultados (AENOR, 2006).

Por otro lado, la poca conciencia generada sobre la importancia de la I+D+i, se encuentra muy ligada con el compromiso que tiene la dirección en éste sentido, debiendo ser superada esta debilidad, ya que puede constituir una barrera para la ejecución de estas actividades. Si bien tomar buenas decisiones estratégicas es una de las mayores responsabilidades del dueño o director general de una 
organización, tanto los empleados como los gerentes deben participar en formular, implementar y evaluar las estrategias (López, 2007).

Tabla 4 - Resultados de la evaluación de la categoría: Recursos Humanos.

\begin{tabular}{l|c|c|c}
\hline Aspecto & $\begin{array}{c}\text { Excelente/ } \\
\text { Bueno } \\
\%\end{array}$ & $\begin{array}{c}\text { Suficiente } \\
\%\end{array}$ & $\begin{array}{c}\text { Insuficiente/ } \\
\text { Deficiente } \\
\%\end{array}$ \\
\hline $\begin{array}{l}\text { Promover la toma de conciencia de la importancia de la } \\
\text { I+D+i }\end{array}$ & 22.22 & 22.22 & 55.56 \\
\hline Motivar al personal & 44.44 & 22.22 & 33.33 \\
\hline Impulsar la participación de todo el personal & 29.63 & 25.93 & 44.44 \\
\hline Fomentar la creatividad y el trabajo en equipo & 37.04 & 29.63 & 33.33 \\
\hline $\begin{array}{l}\text { Simplificar y facilitar los aportes de información de los } \\
\text { distintos departamentos }\end{array}$ & 29.63 & 33.33 & 37.04 \\
\hline Fuen Prapar
\end{tabular}

Fuente: Preparado por los autores

En el gráfico 3 se observan los dos aspectos con menor valoración media en esta categoría: la promoción de la toma de conciencia y el impulso a la participación de todo el personal. La media del resto de ítems se encuentra por encima del nivel suficiente, es decir, fueron valorados en su mayoría como excelentes, buenos o suficientes.

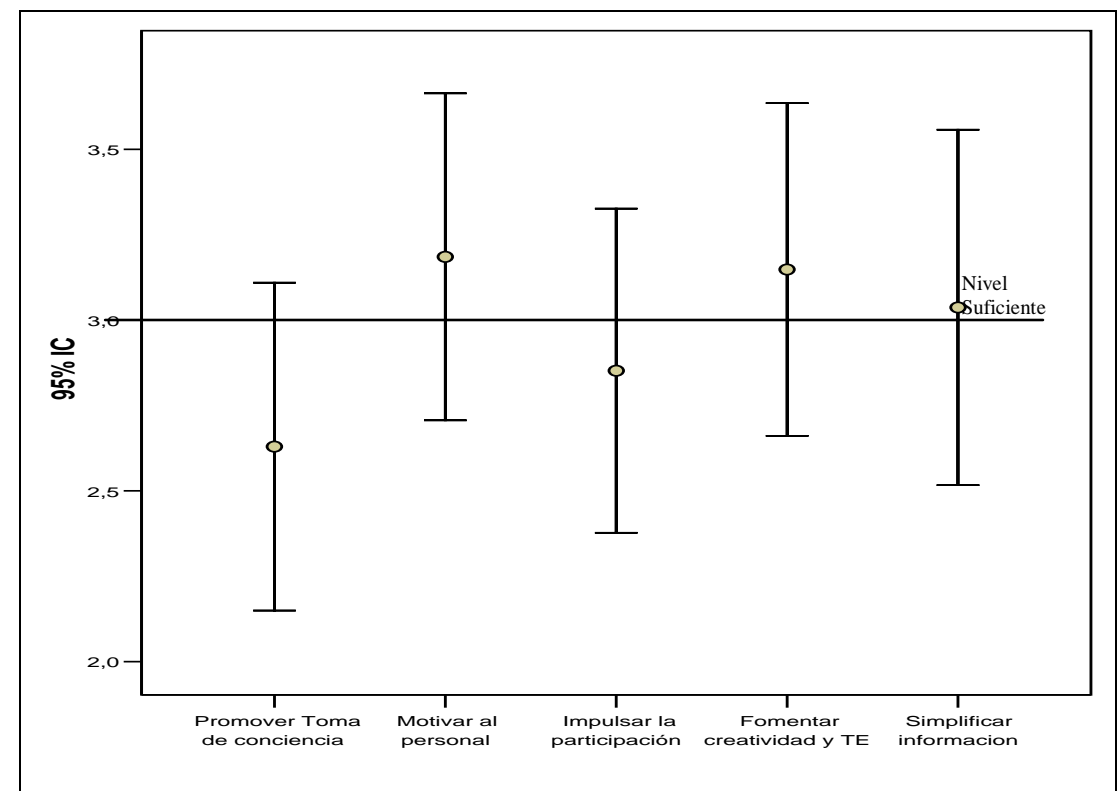

Gráfico 3 - Medias de los ítems correspondientes a: Recursos Humanos.

Fuente: Preparado por los autores. 
Considerando que los recursos humanos de una industria y las políticas que esta establece para gestionarlos son clave para desarrollar ventajas competitivas sostenibles, debido a que las personas y la forma en que se relacionan son muy difíciles de imitar, la innovación abierta promociona el uso de personal con gran talento, tanto interno como externo, que evidentemente no todo trabajará dentro del ámbito de estas industrias.

Se deriva entonces, que se debe promover la toma de conciencia de la importancia de la innovación abierta para lograr favorecer a este sector industrial, a todos los niveles de la organización.

\subsubsection{Actividades de I+D+i.}

Las actividades de $\mathrm{I}+\mathrm{D}+\mathrm{i}$ se consolidan para ejecutar los proyectos, generando conocimiento y desarrollando tecnología o mejorando la actual. En este sentido se evidenció que en las industrias objeto de estudio existe la reflexión para detectar nuevas ideas que permitan guiar el desarrollo de futuros productos y/o procesos, por ello la previsión tecnológica ha sido bien valorada. Al mismo tiempo, se analiza la situación interna y el entorno (sector empresarial), comparando ambas realidades, para poder tomar decisiones al respecto.

No obstante, no se realiza de manera sistemática la captura, el análisis, la difusión y la explotación de informaciones científicas útiles para la organización, es decir, no se lleva a cabo la vigilancia tecnológica, en un sentido estricto. Dentro de las acciones emprendidas prioritariamente por las industrias estudiadas, se encuentran la transferencia de tecnología, considerando tanto la tecnología propia como la posibilidad de incorporar tecnología ajena. Este aspecto es reforzado, por Terán Rojas y Bucci Peluso (2007) cuando señalan que en este sector de empresas el origen de la tecnología proviene de la casa matriz, empresas relacionadas y/o de los proveedores de los equipos.

El producto I+D+i, se fundamenta principalmente en la concreción de ideas en un diseño básico y en rediseñar, demostrar (en real) y producir.

Tabla 5 -Resultados de la evaluación de la categoría: Actividades de I+D+i

\begin{tabular}{l|c|c|c}
\hline Aspecto & $\begin{array}{c}\text { Excelente/ } \\
\text { Bueno } \\
\%\end{array}$ & $\begin{array}{c}\text { Suficiente } \\
\%\end{array}$ & $\begin{array}{c}\text { Insuficiente/ } \\
\text { Deficiente } \\
\%\end{array}$ \\
\hline a) Subcategoría herramientas & \multicolumn{4}{|l}{} \\
\hline Utilizar vigilancia tecnológica & 18.52 & 22.22 & 48.15 \\
\hline Utilizar previsión tecnológica & 48.15 & 22.22 & 29.63 \\
\hline Utilizar la creatividad & 33.33 & 29.63 & 37.04 \\
\hline Utilizar el análisis externo e interno & 40.74 & 29.63 & 29.63 \\
\hline
\end{tabular}

Revista de Administração e Inovação, São Paulo, v. 8, n. 2, p. 05-28, abr./jun. 2011 


\begin{tabular}{|c|c|c|c|}
\hline \multicolumn{4}{|l|}{ b) Subcategoría acciones } \\
\hline Identificar y analizar los problemas y oportunidades & 22.22 & 25.93 & 51.85 \\
\hline Analizar y seleccionar las ideas de $\mathrm{I}+\mathrm{D}+\mathrm{i}$ & 37.04 & 29.63 & 29.63 \\
\hline Planificar, seguir y controlar la cartera de proyectos & 29.63 & 37.04 & 25.93 \\
\hline Utilizar la transferencia de tecnología & 51.85 & 22.22 & 22.22 \\
\hline \multicolumn{4}{|l|}{ c) Subcategoría producto I+D+i } \\
\hline Concretar las ideas en un diseño básico & 51.85 & 14.81 & 29.63 \\
\hline Completar los detalles del diseño antes de las pruebas & 37.04 & 33.33 & 22.22 \\
\hline Crear un prototipo (prueba piloto) & 44.44 & 25.93 & 25.93 \\
\hline Rediseñar, demostrar (en real) y producir & 55.56 & 22.22 & 18.52 \\
\hline Comercializar & 22.22 & 44.44 & 29.63 \\
\hline Documentar todos los cambios durante el proceso & 44.44 & 18.52 & 37.04 \\
\hline \multicolumn{4}{|l|}{ d) Subcategoría: compras } \\
\hline $\begin{array}{l}\text { Asegurarse que los proveedores satisfacen las necesidades del } \\
\text { departamento de I+D+i }\end{array}$ & 44.44 & 18.52 & 37.04 \\
\hline $\begin{array}{l}\text { Verificar las compras para garantizar que se cumple con los } \\
\text { requisitos especificados }\end{array}$ & 66.67 & 7.41 & 25.93 \\
\hline \multicolumn{4}{|l|}{ e) Subcategoría resultados del proceso de I+D+i } \\
\hline Documentar los resultados del proceso de I+D+i & 29.63 & 25.93 & 44.44 \\
\hline Proteger y explotar los resultados de las actividades de I+D+i. & 29.63 & 22.22 & 48.15 \\
\hline
\end{tabular}

Fuente: Preparado por los autores.

En las industrias estudiadas, la tendencia en la subcategoría compras corresponde a la verificación de las compras para garantizar que cumplen los requisitos (74.08 por ciento) y en menor proporción a evaluar a los proveedores (62.96 por ciento). Se observa también, que en estas industrias no se tiende ni a documentar los resultados del proceso ni a proteger y explotar los resultados de las actividades de $\mathrm{I}+\mathrm{D}+\mathrm{i}$, hecho que puede suscitarse a raíz de que no existe un departamento encargado para tal fin.

Al proyectar la media de los ítems valorados en la categoría correspondiente a las actividades de $\mathrm{I}+\mathrm{D}+\mathrm{i}$, se aprecia en el gráfico 4 que se encuentran mejor posicionadas la verificación de las compras y la comercialización y por el contrario el análisis y la selección de las ideas, al igual que la protección y explotación de los resultados son las menos favorecidas. Asimismo, el 61.1 por ciento de los ítems auscultados se encuentran por encima del nivel suficiente. 

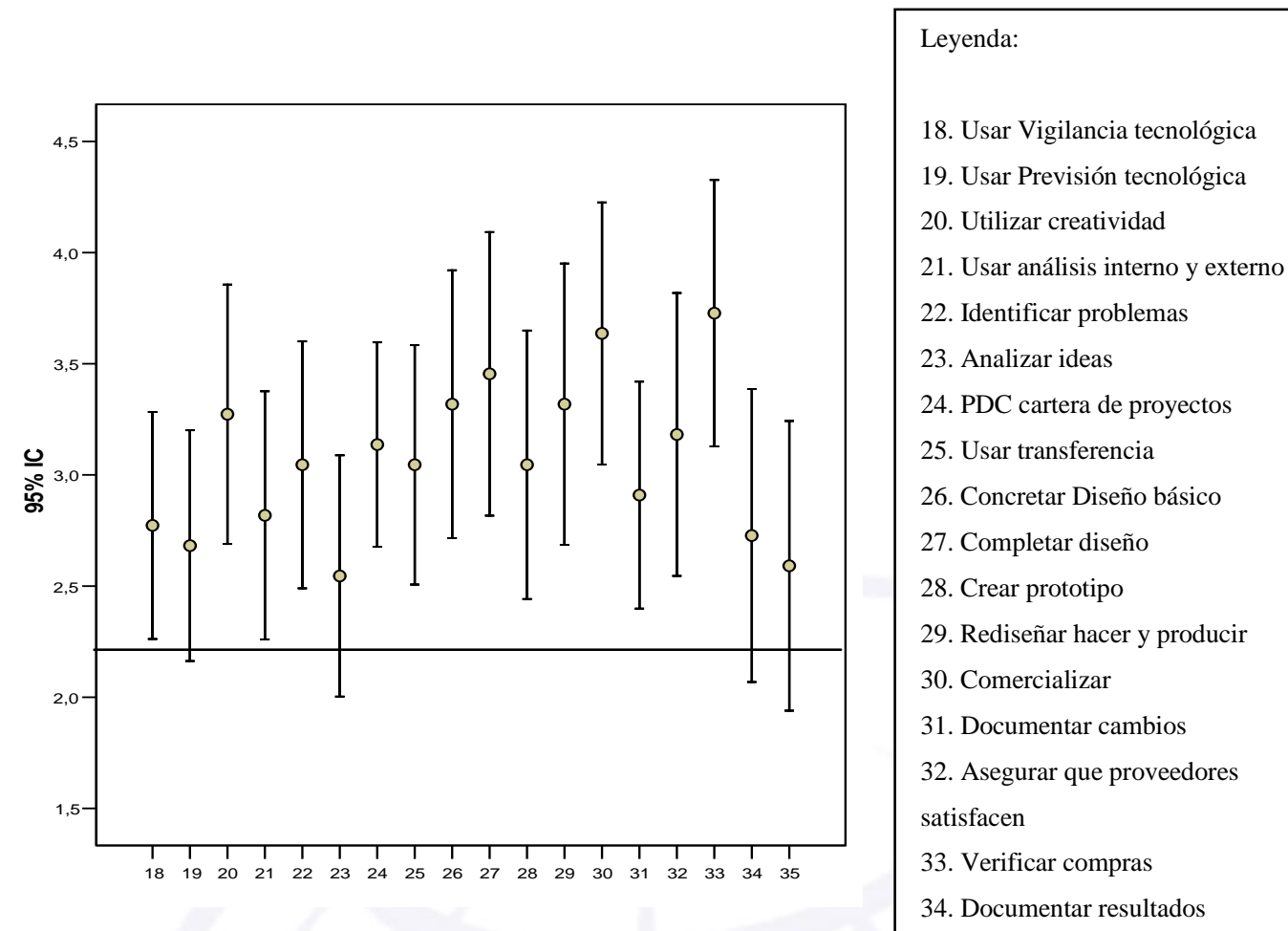

Gráfico 4 - Medias de los ítems correspondientes a: Actividades de I+D+i. Fuente: Preparado por los autores.

Finalmente, se tiene que la categoría mejor valorada, fue la categoría dos, referente a enfoque a las partes interesadas, es decir, que los gerentes y/o directores consideran que se analizan y son atendidas todas las partes interesadas, aunque en menor grado las innovaciones y cambios tecnológicos requeridos por el mercado. Ver gráfico 5, donde se aprecian las medias de todos los ítems objeto de estudio.

Por el contrario, la categoría uno resultó con la menor valoración y corresponde al compromiso de la dirección con el desarrollo e implantación del sistema de gestión de $\mathrm{I}+\mathrm{D}+\mathrm{i}$, a pesar de considerarse de suma importancia para la sobrevivencia y crecimiento de las industrias (Terán Rojas \& Bucci Peluso, 2007).

De igual modo, el 54.28 por ciento de los ítems evaluados son considerados suficientes, buenos o excelentes, es decir, que se practican en las industrias objeto de estudio, en tanto que el 45.71 por ciento son estimados como insuficientes o deficientes. 


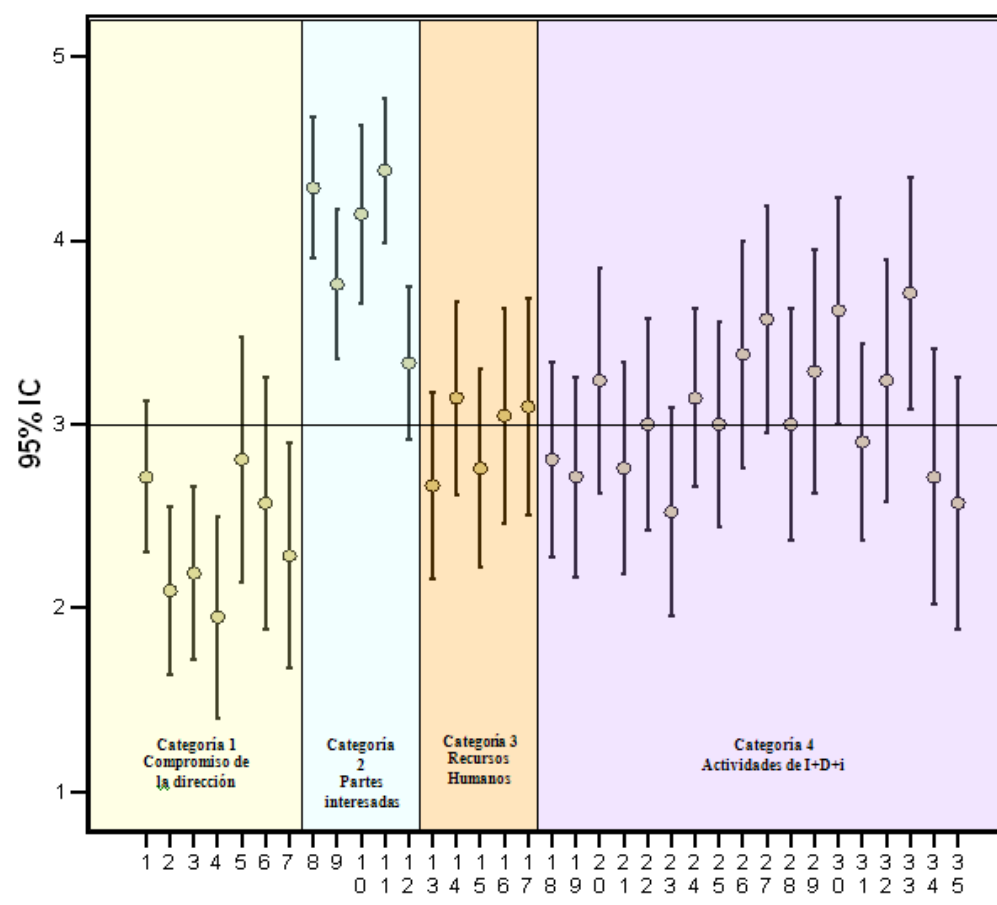

LEYENDA

1. Comunicar importancia

2. Establecer Política I+D+i

3. Establecer Objetivos $\mathrm{I}+\mathrm{D}+\mathrm{i}$

4. Dpto I+D+i

5. Disp de recursos materiales

6. Presupuesto $\mathrm{I}+\mathrm{D}+\mathrm{i}$

7. Politica de proteccion

8. Atender proveedor

9. Atender empleados

10. Atender dueños

11. Atender reglas

12. Atender mercado (innovaciones)

13. Promover Toma de conciencia

14. Motivar al personal

15. Impulsar la participación

16. Fomentar creatividad y TE

17. Simplificar informacion

18. Usar Vigilancia tecnológica

19. Usar Previsión tecnológica

20. Utilizar creatividad

21. Usar análisis interno y externo

22. Identificar problemas

23. Analizar ideas

24. PDC cartera de proyectos

25. Usar transferencia

26. Concretar Diseño básico

27. Completar diseño

28. Crear prototipo

29. Rediseñar hacer y producir

30. Comercializar

31. Documentar cambios

32. Asegurar que proveedores satisfacen

33. Verificar compras

34. Documentar resultados

35. Proteger resultados

Gráfico 5 - Medias de los ítems correspondientes a: compromiso de la dirección enfoques a las partes interesadas, Recursos Humanos, Actividades de I+D+i.

Fuente: Preparado por los autores. 
Ahora bien, la aplicación de la innovación abierta a las PYMIs del sector metalmecánico estaría igualmente sujeta a varias consideraciones, tomando en cuenta los resultados de esta y otras investigaciones en este sector industrial, entre ellas:

- En primer lugar, la apertura hacia la innovación. Ya que en los estudios se ha evidenciado la falta de compromiso a nivel gerencial, en las PYMIs metalmecánicas, y aunque muchos ejecutivos son conscientes de que la innovación es la vía clave para el crecimiento, persisten dudas sobre invertir en procesos que tienen altos costos y gran incertidumbre. Y tal como lo expone Romero Hernández, Muñoz Negrón y Romero Hernández (2006), cuando algunos ejecutivos están dispuestos a apoyar programas de innovación, falta gente con habilidades y los marcos conceptuales adecuados.

- En segundo lugar, con respecto a la motivación del personal para innovar. Las industrias analizadas revelaron motivar al personal y fomentar la creatividad y el trabajo en equipo en un $62 \%$ de manera apropiada, hechos que favorecen el proceso de I+D+i y que puede ser aprovechado para establecer los cimientos para una cultura de innovación abierta.

- En tercer lugar, el aprovechamiento del mercado se enfoca principalmente en el flujo de información que proporcionan los proveedores. De hecho, el origen de la tecnología en el $67 \%$ de las empresas proviene de la casa matriz o alguna empresa relacionada y el $56 \%$ de los proveedores de los equipos. Podría entonces explotarse el flujo de información proveniente del mercado, que tiene una gran importancia para este proceso.

- En cuarto lugar, con respecto al acercamiento al mundo de la investigación el 59\% de las industrias investigadas manifestaron tener vinculaciones técnicas con otras empresas y universidades. Situación que se considera favorable para la innovación abierta y que podría reforzarse en cuanto a la direccionalidad de los proyectos comunes.

- En quinto lugar, la forma en que se mueven, hablan y escuchan, a través del conocimiento de libre acceso y para la observación del entorno se tiene que el 56\% de las empresas están suscritas a publicaciones técnicas y el 78\% han asistido a eventos en los últimos cinco años (Terán Rojas \& Bucci Peluso, 2007). 
- En sexto lugar, se plantea la colaboración, la cual se sitúa entre los mayores desafíos para estas industrias, a saber: el $56 \%$ de los entrevistados señala establecer alianzas de cooperación con otras empresas y formar a los gerentes o supervisores en el tema y un $45 \%$ indica como reto formalizar la tarea de $\mathrm{I}+\mathrm{D}+\mathrm{i}$, además de crear una mejor relación con el entorno científico y tecnológico. Para las micro, pequeñas y medianas industrias es de suma importancia establecer métodos de comunicación eficientes con otras empresas y con instituciones públicas de investigación para superar algunos de los obstáculos a los que se enfrentan, por su naturaleza, tal como la poca capacidad de inversión en I+D+i (Terán Rojas \& Bucci Peluso, 2007).

\section{CONCLUSIONES}

- El sector metalmecánico es fundamental para el desarrollo de las economías de los países, por ser transformador de materias primas, pero en Venezuela ha sido poco desarrollado, tal como se ha evidenciado en otros estudios señalados (Armenti (2006); Bravo et al. (2008); Terán Rojas y Bucci Peluso (2007) y los resultados de esta investigación así lo corroboran.

- La innovación abierta es un paradigma que pretende abrir una organización a la generación de espacios de colaboración, lo cual favorece a las pequeñas y medianas industrias, ya que les permite ser más rápidas y competitivas.

- Un elemento fundamental para la incorporación del paradigma de la innovación abierta en las PYMIs es que exista un compromiso con esta tarea, principalmente a nivel de la gerencia, ya que esto representa un elemento clave para lograr llevar a cabo este proceso con mayor éxito, lo que les permitirá desarrollar ventajas competitivas, además que es un aspecto para valorar la excelencia organizacional de referencia mundial.

- Mejorar los canales de comunicación con el entorno ayudaría al fortalecimiento de la implantación del nuevo paradigma. Además, se mejoraría el ambiente interno y sus relaciones de cooperación, de integración, de interrelación y de trabajo en común. 
Adicionalmente, se comprende mejor el entorno, conociendo más profundamente a los clientes y a los proveedores aprendiendo de otros que realizan actividades similares.

- Consolidar la cultura de innovación es relevante para este proceso y se puede impulsar potenciando el trabajo en equipo, la motivación y la capacitación de los recursos humanos, con la finalidad de incrementar su grado de satisfacción y de confianza y consecuentemente, la eficiencia productiva y competitividad de la industria.

- El proceso de implantación del paradigma de la innovación abierta debe ser gradual y sin ninguna estructura rígida. Pero es importante tomar en cuenta las características propias y las necesidades particulares de cada industria, para lograr un proceso eficaz y fluido.

\section{REFERENCIAS}

Álvarez García, A. (2009). La innovación abierta: ideas de Chesbrough y Von Hippel, Disponible en: http://sociedaddelainformacion.telefonica.es/jsp/articulos/detalle.jsp?elem=8204

Arias, D. (2008). Aplicar la innovación abierta a la empresa. Disponible en: http://www.naider.com/ateneo/articulo_blog.asp?id=313

Armenti, P. (2006). El Sector metalmecánico en el área ACCA. Caracas: Cavenit.

Asheim, B. (2009). La política regional de innovación de la próxima generación: cómo combinar los enfoques del impulso por la ciencia y por el usuario en los sistemas regionales de innovación. Ekonomiaz, 70(1), 106-131.

Asociación Española de Normalización. (2006). Normas UNE 166002 Gestión de la I+D+i: Requisitos del Sistema de Gestión de la $I+D+i$. Madrid: España.

Ayneto, X. (2007). Respondiendo al cambio: la práctica eficaz de la innovación. Mallorca: Cercle D’Economia.

Balestrini, M. (2006). Como se elabora el proyecto de investigación (7 ed.). Caracas. B.L. Consultores Asociados.

Revista de Administração e Inovação, São Paulo, v. 8, n. 2, p. 05-28, abr./jun. 2011 
Bax, L. (2008). La innovación abierta está en boca de todos, pero ¿a qué viene tanto revuelo? Barcelona: Foment del Treball Nacional.

Bravo, M., Suárez, M., Terán, A., \& Sánchez, A. (2008). Caracterización de las PYMES del sector metalmecanico del Municipio Iribarren del Estado Lara. Universidad, Ciencia y Tecnología, 12(49), 279-286.

Chesbrough, H. W. (2009). Innovación abierta: nuevos imperativos para la creación y el aprovechamiento de la tecnologia. Madrid: Plataforma Editorial.

Chesbrough, H. W. (2006). Open innovation: the new imperative for creating and profiting from technology. Boston: Harvard Business School Press.

Consejo Nacional de Promoción de Inversiones. (2006). Apoyo a proyectos metalúrgicos del Gobierno. Disponible en: http://www.conapri.org/articledetails.asp?articleid=232093.

Edwards, M. (2008). Nuevos paradigmas en innovación. Disponible en: http://www.tendencias21.net/innovacion/Nuevos-paradigmas-en-innovacion_a8.html?com.

Frías Rivas, R. (2008). Innovación abierta: una red de redes abierta al mundo. Chile: Universidad del Desarrollo.

García Muñoz, T. (2005). Etapas del proceso investigador: población y muestra. Disponible em: http://www3.unileon.es/dp/ado/ENRIQUE/Diversid/.../poblacionmuestra.doc.

Hidalgo Nuchera, A., Vizán Idoipe, A., \& Torres, M. (2008). Los factores claves de la innovación tecnológica: claves de la competitividad empresarial. Dirección y Organización, 36, 5-22.

Instituto Nacional de Estadística. (2004). Principales indicadores de la industria manufacturera, total 2004. Disponible en: http://www.ine.gob.ve/industria/Indus_Man_2004.htm.

Iturbe-Ormaetxe, J. (2008). Redes sociales e innovación abierta: apuntes críticos. Telos, 76, 114-117.

Longanezi, T., Coutinho, P. L. A., \& Bomtempo, J. V. M. (2008). Um modelo referencial para a prática da inovação. Journal of Technology Management \& Innovation, 3(1), 74-83.

Revista de Administração e Inovação, São Paulo, v. 8, n. 2, p. 05-28, abr./jun. 2011 
López, R. (2007). Ventaja competitiva. Disponible en:

http://pyme.mx/pyme/index.php?option=com_content\&view=article\&id=121:070815-revistapyme-agosto-3\&catid=32:2007\&Itemid $=40$.

Morillo, M. (2005). Análisis de la cadena de valor industrial y de la cadena de valor agregado para la pequeñas y medianas industrias. Actualidad Contable FACES, 8(10), 53-70.

Organización para la Cooperación y el Desarrollo Económicos. (2006). Manual de OSLO: guía para la recogida e interpretación de datos sobre innovación (3 ed.). (J. Zamorano Ogállal, Trad.). Madrid: Grupo Trasga.

Palella, S., \& Martins, F. (2003). Metodología de la investigación cuantitativa. Caracas: Fondo Editorial de la UPEL.

Romero Hernández, O., Muñoz Negrón, D., \& Romero Hernández, S. (2006). Introducción a la ingeniería: un enfoque industrial. México, D. F.: International Thomson.

Sala-i-Martin, X., Blanke, J., Hanouz, M. D., Geiger, T., Mia, I., \& Paua, F. (2007). The global competitiveness index: measuring the productive potential of nations. In M. E. Porter, K. Schwab, \& X. Sala-i-Martin (Eds.), The global competitiveness report 2007-2008 (pp. 3-50). Basingstoke: Palgrave Macmillan.

Terán Rojas, A., \& Bucci Peluso, N. (2007). Evaluación de actividades de I+D e innovación. Caso: empresas metalmecánicas [CD-ROM]. Memorias del Seminario Latino-Iberoamericano de Gestión Tecnológica, 12. Buenos Aires: ALTEC.

Universidad Pedagógica Experimental Libertador. (2006) Manual de trabajos de grado de especialización y maestría y tesis doctorales. Caracas: Fedupel. 


\title{
OPEN INNOVATION AS AN ELEMENT OF ANALYSIS FOR SMALL AND MEDIUM INDUSTRIES. CASE ENGINEERING SECTOR
}

\begin{abstract}
Open innovation is based on the idea that companies are no longer capable of addressing the whole process of innovation through their own resources. It is a paradigm that assumes that organizations can and should use external ideas as well as internal ideas. The objective of this research was to identify management practices in the process of open innovation in small and medium enterprises (SMEs) in emerging economies. The methodology was based on field research and the data collection technique used was the interview, based on a questionnaire (focusing on the Spanish UNE 166002 standards), whose categories were: managerial commitment, focus on stakeholders, human resources and research, and development and innovation. Thirty companies participated in this study, which concluded that a key element for the incorporation of open innovation is managerial commitment to the industrial sector. This has been shown to improve communication channels with the surroundings, and to strengthen the innovation culture through the promotion of teamwork, motivation and the training of human resources as well as through the consideration of both internal and external sources.
\end{abstract}

Keywords: Open Innovation; Metal-mechanic industries; Emergent economies; Small and Medium Enterprises (SME).

Data do recebimento do artigo: 25/01/2011

Data do aceite de publicação: 20/04/2011 